\title{
Rank one strange attractors in periodically kicked Chua's system with time delay
}

\author{
Wenjie Yang ${ }^{1}$, Yiping Lin ${ }^{1 *}$, Yunxian Dai ${ }^{1}$ and Yusheng Jia ${ }^{2}$
}

\section{"Correspondence:}

linyiping689@163.com

'Department of Applied

Mathematics, Kunming University of

Science and Technology, Kunming,

Yunnan 650500, P.R. China

Full list of author information is

available at the end of the article

\begin{abstract}
In this paper, rank one strange attractor in a periodically kicked time-delayed system is investigated. It is shown that rank one strange attractors occur when the delayed system under a periodic forcing undergoes Hopf bifurcation. Our discussion is based on the theory of rank one maps formulated by Wang and Young. As an example, periodically kicked Chua's system with time-delay is considered, conditions for rank one chaos along with the results of numerical simulations are presented.
\end{abstract}

Keywords: rank one strange attractors; time-delayed system; periodically kicked; Chua's circuit

\section{Introduction}

Recently, a chaos theory on rank one maps has been developed by Wang and Young. In 2001, Wang and Oksasoglu [1] gave simple conditions that guarantee the existence of strange attractors with a single direction of instability and certain controlled behaviors. In 2008, Wang and Young accomplished a more comprehensive understanding of the complicated geometric and dynamical structures of a specific class of non-uniformly hyperbolic homoclinic tangles. For certain differential equations, through their well-defined computational process, the existence of the indicated phenomenon of rank one chaos was verified [2]. In 2009, Chen and Han studied the existence of rank one chaos in a periodically kicked planar equation with heteroclinic cycle [3]. In 2012, Fang studied the synchronization between rank-one chaotic systems without and with delay using linear delayed feedback control method [4].

In 1984, Jackson et al. studied Chua's circuit as follows [5]:

$$
\left\{\begin{array}{l}
C_{1} \frac{d v_{C_{1}}}{d t}=G\left(v_{C_{2}}-v_{C_{1}}\right)-g\left(v_{C_{1}}\right) \\
C_{2} \frac{d v_{C_{2}}}{d t}=G\left(v_{C_{1}}-v_{C_{2}}\right)+i_{L} \\
L \frac{d i_{L}}{d t}=-v_{C_{2}}
\end{array}\right.
$$

where $v_{C_{1}}, v_{C_{2}}, i_{L}$ denote voltage across $C_{1}, C_{2}$ and current through $L$, respectively. They reported that a chaotic attractor has been observed in Eq. (1). In [6], Wang et al. considered Eq. (1) as the following form:

$$
\left\{\begin{array}{l}
\frac{d v_{1}}{d t}=\frac{1}{C_{1}}\left(G\left(v_{2}-v_{1}\right)-f\left(v_{1}\right)\right), \\
\frac{d v_{2}}{d t}=\frac{1}{C_{2}}\left(i-\left(G\left(v_{2}-v_{1}\right)\right),\right. \\
\frac{d i}{d t}=-\frac{1}{L}\left(v_{2}+R_{0} i+\varepsilon \sin \left(\sigma v_{1}(t-\tau)\right)\right) .
\end{array}\right.
$$

(c) 2015 Yang et al.; licensee Springer. This is an Open Access article distributed under the terms of the Creative Commons Attribution License (http://creativecommons.org/licenses/by/4.0), which permits unrestricted use, distribution, and reproduction in any medium, provided the original work is properly credited. 
They studied this time-delay Chua's circuit by theoretical analysis, computer simulations and circuit experiments. In [7], Wang and Oksasoglu considered Chua's system of the following form:

$$
\left\{\begin{array}{l}
\dot{x}=a[y-f(x)]+\varepsilon P_{T} x \\
\dot{y}=x-y+z \\
\dot{z}=-b y
\end{array}\right.
$$

where $f(x)$ is chosen to be a cubic function of the form $f(x)=c x+d x^{3}$. They researched rank one strange attractors in this periodically kicked Chua's circuit.

In this paper, we first try to develop rank one theory from an ordinary differential equation to a time-delayed system, then consider Chua's system (3) as the following form:

$$
\left\{\begin{array}{l}
\dot{x}(t)=a\left[y(t)-\left(c x(t)+d x^{3}(t)\right)\right], \\
\dot{y}(t)=x(t)-y(t)+z(t)+\varepsilon P_{T} y(t), \\
\dot{z}(t)=-b y(t-\tau),
\end{array}\right.
$$

where $a, b, c, d$ and $\tau$ are real parameters, $\varepsilon P_{T} y(t)$ is a time-periodic forcing term with $P_{T}=\sum_{n=-\infty}^{\infty} \delta(t-n t)$. The stability of equilibria, the bifurcating of periodic solutions and the rank one chaos of the periodically kicked delayed system are investigated.

This paper is organized as follows. In Section 2, we give preliminaries about the rank one chaotic theory. In Section 3, we derive the rank one chaotic theory for a time-delayed system. In Section 4, we take Chua's system as an example. In Section 5, numerical simulations are presented. Conclusions are given in Section 6.

\section{Preliminaries}

To properly motivate the studies presented in this paper, we first give a brief overview on the studies of rank one strange attractors, which can be constructed in the following way.

Following the work done by Wang and Young in [7], we let $\mathbf{u} \in \mathrm{R}^{m}, m \geq 2$ be the phase variable and $t \in \mathrm{R}$ be the time. Consider the following system of equations:

$$
\frac{d \mathbf{u}}{d t}=\mathbf{A}_{\mu} \mathbf{u}+f_{\mu}(\mathbf{u})+\varepsilon P_{T} \Phi(\mathbf{u}),
$$

where $\mathbf{A}_{\mu}$ is a real $m$ by $m$ matrix, and $f_{\mu}(\mathbf{u})$ is a vector-valued real analytic function in $\mathbf{u}$ defined on a given neighborhood of $\mathbf{u}=\mathbf{0}$ such that $f(\mathbf{0})=\mathbf{0}, D_{\mathbf{u}} f(\mathbf{0})=\mathbf{0}$. Both $\mathbf{A}_{\mu}$ and $f_{\mu}(\mathbf{u})$ are smooth dependents of parameter $\mu$ around $\mu=0$. $\varepsilon$ is a parameter that controls the magnitude of the forcing, $\Phi(\mathbf{u})$ is a real vector-valued function of $\mathbf{u}$ that represents the shape of the forcing and $P_{T}=\sum_{n=-\infty}^{\infty} \delta(t-n T)$. When $\varepsilon=0$, the undisturbed system is

$$
\frac{d \mathbf{u}}{d t}=\mathbf{A}_{\mu} \mathbf{u}+f_{\mu}(\mathbf{u})
$$

Firstly, we give several definitions.

Definition 1 [8] Let $f: M \rightarrow M$ be a diffeomorphism of a compact Riemannian manifold onto itself. We say that $f$ is an Anosov diffeomorphism if the tangent space at every $x \in M$ is split into $E^{u}(x) \oplus E^{s}(x)$, where $E^{u}$ and $E^{s}$ are $D f$-invariant subspaces, $\left.D f\right|_{E^{u}}$ is uniformly 
expanding and $\left.D f\right|_{E^{s}}$ is uniformly contracting. A compact $f$-invariant set $\Lambda \subset M$ is called an attractor if there is a neighborhood $U$ of $\Lambda$ called its basin such that $f^{n} x \rightarrow \Lambda$ for every $x \in U . \Lambda$ is called an Axiom A attractor if the tangent bundle over $\Lambda$ is split into $E^{u} \oplus E^{s}$ as above.

Definition 2 [8] Let $f$ be a $C^{2}$ diffeomorphism with an Axiom A attractor $\Lambda$. Then there is a unique $f$-invariant Borel probability measure $\mu$ on $\Lambda$ that is characterized by each of the following (equivalent) conditions:

(i) $\mu$ has absolutely continuous conditional measures on unstable manifolds;

(ii)

$$
h_{\mu}(f)=\int\left|\operatorname{det}\left(\left.D f\right|_{E^{u}}\right)\right| d \mu,
$$

where $h_{\mu}(f)$ is the metric entropy of $f$;

(iii) there is a set $V \subset U$ having full Lebesgue measure such that for every continuous observable $\varphi: U \rightarrow R$, we have, for every $x \in V$,

$$
\frac{1}{n} \sum_{i=0}^{n-1} \varphi\left(f^{i} x\right) \rightarrow \int \varphi d \mu
$$

(iv) $\mu$ is the zero-noise limit of small random perturbations of $f$.

Then the invariant measure $\mu$ is called the Sinai-Ruelle-Bowen measure, or SRB measure, of $f$.

Definition 3 Given a map $f$, the Lyapunov exponent is defined at $x_{0}$ as follows:

$$
\lambda\left(x_{0}\right)=\lim _{N \rightarrow+\infty} \frac{1}{N} \ln \left|\prod_{i=0}^{N-1} f^{\prime}\left(x_{i}\right)\right|=\lim _{N \rightarrow+\infty} \frac{1}{N} \sum_{i=0}^{N-1} \ln \left|f^{\prime}\left(x_{i}\right)\right|,
$$

where $x_{i}=f\left(x_{i-1}\right)(i=1,2, \ldots, N-1)$.

Now, we consider $(5)_{\varepsilon}$. Assume the following:

(A1) Let $\left\{\lambda_{i}\right\}_{i=1}^{m}$ be the eigenvalues of $\mathbf{A}_{\mu}$. There is a conjugated pair,

$$
\lambda_{1,2}=\alpha(\mu) \pm i \omega(\mu)
$$

such that $\alpha(0)=0, w(0) \neq 0,(d / d \mu) \alpha(0) \neq 0$, and there exists $c>0$ such that $\operatorname{Re}\left(\lambda_{i}\right)<-c$, $i \geq 3$.

Then the flow on the central manifold $\mathrm{W}^{c}$ of system $(5)_{0}$ can be explicitly written by using a complex variable $z$ in the following normal form:

$$
\frac{d z}{d t}=(\alpha(\mu)+i \omega(\mu)) z+k_{1}(\mu) z^{2} \bar{z}+k_{2}(\mu) z^{3} \bar{z}^{2}+\cdots .
$$

Write that

$$
k_{1}(\mu)=-E(\mu)+i F(\mu)
$$

and assume that 
(A2) $E(0)>0$.

Assume that a real linear coordinate change $\mathbf{u} \rightarrow L_{\mu} \mathbf{u}:=(\xi, \eta, \mathbf{w})^{T}$ transfers Eq. (5) ${ }_{\varepsilon}$ into

$$
\left\{\begin{array}{l}
\frac{d \xi}{d t}=\alpha \xi+\omega \eta+f_{\xi}+\varepsilon \Phi_{\xi} P_{T}(t), \\
\frac{d \eta}{d t}=-\omega \xi+\alpha \eta+f_{\eta}+\varepsilon \Phi_{\eta} P_{T}(t), \\
\frac{d \mathbf{w}}{d t}=A^{(s)} \mathbf{w}+\omega \eta+f_{\mathbf{w}}+\varepsilon \Phi_{\mathbf{w}} P_{T}(t),
\end{array}\right.
$$

where $\xi, \eta$ are scalars, $\mathbf{w}$ is an $m-2$-vector, $A^{(s)}$ is an $(m-2)$ by $(m-2)$ matrix of eigenvalues with negative real parts, and

$$
\begin{aligned}
& \left(f_{\xi}, f_{\eta}, f_{\mathbf{w}}\right)^{T}=L_{\mu} f_{\mu}\left(L_{\mu}^{-1}(\xi, \eta, \mathbf{w})^{T}\right), \\
& \left(\Phi_{\xi}, \Phi_{\eta}, \Phi_{\mathbf{w}}\right)^{T}=L_{\mu} \Phi\left(L_{\mu}^{-1}(\xi, \eta, \mathbf{w})^{T}\right) .
\end{aligned}
$$

Let $(\hat{r}, \hat{\mathbf{s}}) \in \mathrm{R}^{+} \times \mathrm{S}^{m-1}$ be such that $(\xi, \eta, \mathbf{w})^{T}=\hat{r} \hat{\mathbf{s}}$, and define

$$
\left(\Psi_{\xi}(\hat{r}, \hat{\mathbf{s}}), \Psi_{\eta}(\hat{r}, \hat{\mathbf{s}}), \Psi_{w}(\hat{r}, \hat{\mathbf{s}})\right)^{T}=\left(\Phi_{\xi}, \Phi_{\eta}, \Phi_{\mathbf{w}}\right)^{T}
$$

Further let $\left\{\hat{\mathbf{s}}_{0}=(\cos \theta, \sin \theta, \mathbf{0}) \in \mathrm{S}^{m-1}, \theta \in[0,2 \pi)\right\}$ be the unit circle in $(\xi, \eta)$-plane in $(\xi, \eta, \mathbf{w})$-space, and define

$$
\varphi(\theta)=\cos \theta \Psi_{\xi}\left(0, \hat{\mathbf{s}}_{0}\right)+\sin \theta \Psi_{\eta}\left(0, \hat{\mathbf{s}}_{0}\right) .
$$

The time- $T$ map of Eq. (5) $)_{\varepsilon}$ is denoted by $F_{\mu, \varepsilon, T}$, where $\mu$ is the bifurcation parameter of the unperturbed Eq. (5) $)_{0}$ and $\varepsilon, T$ are the parameters of forcing. Assume that

(a) (A1)-(A2) hold for Eq. (5) ${ }_{\varepsilon}$;

(b) $\varphi(\theta)$ in Eq. (10) is a Morse function; and

(c) $\mu, \varepsilon$ are such that $0<\mu \ll 1,0<\varepsilon \ll 1$.

The following theorem is obtained by Wang and Oksasoglu [9].

Theorem 1 Let the values of $\mu$ and $\varepsilon$ be fixed and assume that (a)-(c) hold. Regard the period $T$ of the forcing as a parameter and define $F_{T}=F_{\mu, \varepsilon, T}$. Then there exists a constant $K_{1}$, determined exclusively by $\varphi(\theta)$, such that if

$$
\left|\varepsilon \frac{F(0)}{E(0)}\right|>K_{1}
$$

then there exists a positive measure set $\Delta \subset\left(\mu^{-1}, \infty\right)$ for $T$ so that for $T \in \Delta, F_{T}$ has a strange attractor $\Lambda$ admitting no periodic sinks. This is to say that there exists an open neighborhood $U$ of $\Lambda$ in $R^{m}$ such that $F_{T}$ has a positive Lyapunov exponent for Lebesgue almost every point in $U$. Furthermore, $F_{T}$ admits an ergodic SRB measure, with respect to which almost every point of $U$ is generic.

\section{Rank one strange attractors of a delayed system}

In this section we consider the nonlinear delayed differential equation

$$
\frac{d \mathbf{u}}{d t}=L_{\mu} \mathbf{u}_{t}+f_{\mu}\left(\mathbf{u}_{t}\right)+\varepsilon P_{T} \Phi\left(\mathbf{u}_{\mathbf{t}}\right)
$$


where $\mathbf{u}_{t}(\theta)=\mathbf{u}(t+\theta), \theta \in[-r, 0]$ for $r>0, L_{\mu}: C[-r, 0] \rightarrow \mathrm{R}^{n}$ is a linear operator, $f_{\mu}$ : $\mathrm{C}[-r, 0] \rightarrow \mathrm{R}^{n}$ is a nonlinear term satisfying $f_{\mu}(\mathbf{0})=\mathbf{0}, D_{\mathbf{u}} f_{\mu}(\mathbf{0})=\mathbf{0} . f_{\mu}$ and $L_{\mu}$ depend on $\mu$ analytically for $|\mu|$ is sufficiently small, and $P_{T}=\sum_{n=-\infty}^{\infty} \delta(t-n T)$. When $\varepsilon=0$, the undisturbed system is

$$
\frac{d \mathbf{u}}{d t}=L_{\mu} \mathbf{u}_{t}+f_{\mu}\left(\mathbf{u}_{t}\right)
$$

For the linear system $\dot{\mathbf{u}}=L_{\mu} \mathbf{u}_{t}$, there is an $n \times n$ matrix $\eta(\cdot, \mu):[-r, 0] \rightarrow \mathrm{R}^{n^{2}}$ such that for any $\varphi \in \mathrm{C}[-r, 0]$

$$
L_{\mu} \phi=\int_{-r}^{0} d \eta(\theta, \mu) \phi(\theta) .
$$

Let the spectral set of $L_{\mu}$

$$
\sigma(\mu)=\left\{\lambda \mid \operatorname{det}\left(\lambda I-L_{\mu} e^{\lambda \theta} I\right)=0\right\}
$$

satisfy the following:

(B1) There is a simple conjugated pair

$$
\lambda_{1,2}=a(\mu) \pm i \omega(\mu)
$$

such that $a(0)=0, \omega(0)=\omega_{0}>0,(d / d \mu) a(0) \neq 0$, and there exists $c>0$ such that for any $\lambda \in \sigma(\mu), \lambda \neq \lambda_{1,2}, \operatorname{Re}\left(\lambda_{i}\right)<-c, i \geq 3$.

Then the flow on the central manifold $\mathrm{W}^{c}$ of system $(11)_{0}$ can be written by using Hassard's method [10] in the following normal form:

$$
\dot{z}=i w_{0} z+g(z, \bar{z})=i w_{0} z+\frac{g_{20}}{2} z^{2}+g_{11} z \bar{z}+\frac{g_{02}}{2} \bar{z}^{2}+\frac{g_{21}}{2} z^{2} \bar{z}+\cdots
$$

at $\mu=0$. Assume that $g_{20}=g_{11}=g_{02}=0$. We write $\frac{g_{21}}{2}$ as

$$
\frac{g_{21}}{2}=-E(0)+i F(0) \text {. }
$$

Suppose that

(B2) $E(0)>0$.

Then we know that system $(11)_{0}$ has a supercritical Hopf bifurcation near the equilibrium.

We define for $\phi \in \mathrm{C}[-r, 0]$

$$
A(\mu) \phi= \begin{cases}\frac{d \phi}{d \theta}, & \theta \in[-r, 0), \\ \int_{-r}^{0} d \eta(s, \mu) \phi(s)=L_{\mu} \phi, & \theta=0,\end{cases}
$$

and

$$
R \phi= \begin{cases}0, & \theta \in[-r, 0), \\ f_{\mu}(\phi)+\varepsilon P_{T} \Phi(\phi), & \theta=0 .\end{cases}
$$


Since $\frac{d \mathbf{u}_{t}}{d \theta}=\frac{d \mathbf{u}_{t}}{d t}$, Eq. $(11)_{\varepsilon}$ becomes

$$
\dot{\mathbf{u}}_{t}=A(\mu) \mathbf{u}_{t}+R \mathbf{u}_{t}
$$

For $\theta=0$ Eq. (17) is Eq. (11) $)_{\varepsilon}$.

Following Hassard's method, we know that $z$ satisfies the equation

$$
z(t)=\left\langle q^{*}(\theta), u_{t}(\theta)\right\rangle
$$

where $q^{*}(\theta)$ is an eigenvector corresponding to the eigenvalue $-i \omega_{0}$ of $A^{*}$ which is the adjoint operator of $A(0)$.

When $\mu=0$, let $A=A(0)$ and we define

$$
\begin{aligned}
W(t, \theta) & =u_{t}(\theta)-z(t) q(\theta)-\bar{z}(t) \bar{q}(\theta) \\
& =u_{t}(\theta)-2 \operatorname{Re}\{z(t) q(\theta)\} \\
& =W(z(t), \bar{z}(t), \theta) .
\end{aligned}
$$

Then from Eq. $(11)_{\varepsilon}$ and the definitions of $A, A^{*}$ we obtain

$$
\begin{aligned}
\dot{z} & =\left\langle q^{*}(\theta), \dot{u}_{t}(\theta)\right\rangle \\
& =\left\langle q^{*}(\theta), A u_{t}(\theta)+R u_{t}(\theta)\right\rangle \\
& =\left\langle q^{*}(\theta), A u_{t}(\theta)\right\rangle+\left\langle q^{*}(\theta), R u_{t}(\theta)\right\rangle \\
& =\left\langle A^{*} q^{*}(\theta), u_{t}(\theta)\right\rangle+\left\langle q^{*}(\theta), R u_{t}(\theta)\right\rangle \\
& =i \omega_{0}\left\langle q^{*}(\theta), u_{t}(\theta)\right\rangle+\left\langle q^{*}(\theta), R u_{t}(\theta)\right\rangle .
\end{aligned}
$$

At $\theta=0$,

$$
\begin{aligned}
\dot{z} & =i \omega_{0} z+\bar{q}^{*}(0) f_{0}(W(z, \bar{z}, 0)+2 \operatorname{Re} z q(0))+\varepsilon P_{T} \bar{q}^{*}(0) \Phi(W(z, \bar{z}, 0)+2 \operatorname{Re} z q(0)) \\
& =i \omega_{0} z+g(z, \bar{z})+\varepsilon P_{T} \bar{q}^{*}(0) \Phi(W(z, \bar{z}, 0)+2 \operatorname{Re} z q(0)) \\
& =i \omega_{0} z+(-E(0)+i F(0)) z^{2} \bar{z}+\cdots+\varepsilon P_{T} \bar{q}^{*}(0) \Phi(W(z, \bar{z}, 0)+2 \operatorname{Re} z q(0)) .
\end{aligned}
$$

We can calculate

$$
\dot{W}=\dot{u}_{t}-\dot{z} q-\dot{\bar{z}} \bar{q}=A W+H(z, \bar{z}, 0)
$$

Then Eq. (17) can be written as

$$
\left\{\begin{array}{l}
\dot{z}=i \omega_{0} z+(-E(0)+i F(0)) z^{2} \bar{z}+\cdots+\varepsilon P_{T} \bar{q}^{*}(0) \Phi(W(z, \bar{z}, 0)+2 \operatorname{Re} z q(0)), \\
\dot{W}=A W+H(z, \bar{z}, 0)
\end{array}\right.
$$

Let $W \in \mathcal{B}$, where $\mathcal{B}$ is a Banach space, let $z=x+i y$ in (21), and define

$$
\begin{aligned}
& \Psi_{x}(x, y)=\operatorname{Re}\left\{\bar{q}^{*}(0) \Phi(W(x, y, 0)+2 \operatorname{Re}(x+i y) q(0))\right\} \\
& \Psi_{y}(x, y)=\operatorname{Im}\left\{\bar{q}^{*}(0) \Phi(W(x, y, 0)+2 \operatorname{Re}(x+i y) q(0))\right\} .
\end{aligned}
$$


We further let $x=\cos \theta, y=\sin \theta, \mathbf{W}=\mathbf{0}$ in (22), then $\left\{\hat{\mathbf{s}}_{0}=(\cos \theta, \sin \theta, \mathbf{0}) \in S \times \mathcal{B}, \theta \in\right.$ $[0,2 \pi)\}$ is the unit circle in $(x, y)$-plane in $(x, y, \mathbf{W})$-space. Define

$$
\phi(\theta)=\cos \theta \Psi_{x}\left(\hat{s}_{0}\right)+\sin \theta \Psi_{y}\left(\hat{s}_{0}\right) .
$$

The time- $T$ map of Eq. $(11)_{\varepsilon}$ is denoted by $F_{\mu, \varepsilon, T}$, where $\mu$ is the bifurcation parameter of the unperturbed Eq. (11) $)_{0}$ and $\varepsilon, T$ are the parameters of forcing. Assume that

(a) (B1)-(B2) hold for Eq. (11)

(b) $\phi(\theta)$ in Eq. (23) is a Morse function; and

(c) $\mu$, $\varepsilon$ are such that $0<\mu \ll 1,0<\varepsilon \ll 1$.

Then we obtain the following.

Theorem 2 Let the values of $\mu$ and $\varepsilon$ be fixed and assume that (a)-(c) hold. Regard the period $T$ of the forcing as a parameter and define $F_{T}=F_{\mu, \varepsilon, T}$. Then there exists a constant $K_{2}$, determined exclusively by $\phi(\theta)$, such that if

$$
\left|\varepsilon \frac{F(0)}{E(0)}\right|>K_{2}
$$

then there exists a positive measure set $\Delta \subset\left(\mu^{-1}, \infty\right)$ for $T$ so that for $T \in \Delta, F_{T}$ has a strange attractor $\Lambda$ admitting no periodic sinks. This is to say that there exists an open neighborhood $U$ of $\Lambda$ such that $F_{T}$ has a positive Lyapunov exponent for Lebesgue almost every point in $U$. Furthermore, $F_{T}$ admits an ergodic SRB measure, with respect to which almost every point of $U$ is generic.

Proof We can easily see that (B1), (B2) in Theorem 2 correspond to (A1), (A2) in Theorem 1. After transformations, Eq. (23) in $(x, y)$-plane corresponds to Eq. (10) in $(\xi, \eta)$-plane, they are on the central manifolds. Obviously the conditions in Theorem 1 are satisfied.

\section{Analysis of rank one strange attractors in delayed Chua's system}

In this section, we consider Eq. $(4)_{\varepsilon}$. The corresponding undisturbed system is as follows:

$$
\left\{\begin{array}{l}
\dot{x}(t)=a\left[y(t)-\left(c x(t)+d x^{3}(t)\right)\right], \\
\dot{y}(t)=x(t)-y(t)+z(t), \\
\dot{z}(t)=-b y(t-\tau) .
\end{array}\right.
$$

It always has equilibria $E_{i}^{*}\left(x_{i}^{*}, y_{i}^{*}, z_{i}^{*}\right), i=1,2,3$, where

$$
\begin{aligned}
& x_{1}^{*}=0, \quad y_{1}^{*}=0, \quad z_{1}^{*}=0, \\
& x_{2}^{*}=\sqrt{\frac{-c}{d}}, \quad y_{2}^{*}=0, \quad z_{2}^{*}=-x_{2}^{*}, \\
& x_{3}^{*}=-\sqrt{\frac{-c}{d}}, \quad y_{3}^{*}=0, \quad z_{3}^{*}=-x_{2}^{*} .
\end{aligned}
$$

Let $E^{*}=\left(x^{*}, y^{*}, z^{*}\right)$ be an arbitrary equilibrium, and let $\bar{x}(t)=x(t)-x^{*}, \bar{y}(t)=y(t)-y^{*}$, $\bar{z}(t)=z(t)-z^{*}$, still denote $\bar{x}(t), \bar{y}(t), \bar{z}(t)$ by $x(t), y(t), z(t)$, respectively, then the linearized 
system of the corresponding equation at $E^{*}$ is as follows:

$$
\dot{u}=A u(t)+B u(t-\tau),
$$

where $u(t)=[x(t), y(t), z(t)]^{T}$,

$$
A=\left[\begin{array}{ccc}
-a c-3 a d x^{* 2} & a & 0 \\
1 & -1 & 1 \\
0 & 0 & 0
\end{array}\right], \quad B=\left[\begin{array}{ccc}
0 & 0 & 0 \\
0 & 0 & 0 \\
0 & -b & 0
\end{array}\right]
$$

We consider the following cases.

(1) $E^{*}=E_{1}^{*}$

The characteristic equation of system (24) is

$$
\lambda^{3}+\alpha_{1} \lambda^{2}+\alpha_{2} \lambda+b e^{-\lambda \tau}\left(\lambda+\beta_{1}\right)=0,
$$

where $\alpha_{1}=a c+1, \alpha_{2}=a c-a, \beta_{1}=a c$.

When $\tau=0$, Eq. (25) becomes

$$
\lambda^{3}+\alpha_{1} \lambda^{2}+\left(\alpha_{2}+b\right) \lambda+b \beta_{1}=0
$$

The Routh-Hurwitz criterion implies that if

$$
\left(\mathrm{H}_{1}\right) \quad a c>-1, \quad a b c>0, \quad \text { and } \quad b+a(c-1)(a c+1)>0 \text {, }
$$

then all roots of Eq. (26) have negative real parts.

Suppose that $i \omega(\omega>0)$ is a root of Eq. (25) in the imaginary axis. Substituting it to Eq. (25) and separating the real and imaginary parts, we have

$$
\left\{\begin{array}{l}
b \omega \cos (\omega \tau)-b \beta_{1} \sin (\omega \tau)=\omega^{3}-\alpha_{2} \omega, \\
b \omega \sin (\omega \tau)+b \beta_{1} \cos (\omega \tau)=\alpha_{1} \omega^{2},
\end{array}\right.
$$

which is equivalent to

$$
\omega^{6}+e_{1} \omega^{4}+e_{2} \omega^{2}+e_{3}=0 .
$$

Let $z=\omega^{2}$, then Eq. (28) becomes

$$
G(z)=z^{3}+e_{1} z^{2}+e_{2} z+e_{3}=0,
$$

where $e_{1}=\alpha_{1}^{2}-2 \alpha_{2}, e_{2}=\alpha_{2}^{2}-b^{2}, e_{3}=-b^{2} \beta_{1}^{2}$.

Since $G(0)=-b^{2} \beta_{1}^{2}<0, G(+\infty)=+\infty$, we immediately obtain the following.

Lemma $1 E q$. (29) has at least one positive root since $e_{3}<0$.

We assume that Eq. (29) has three positive roots, $z_{1}, z_{2}, z_{3}$, and $\omega_{1}=\sqrt{z_{1}}, \omega_{2}=\sqrt{z_{2}}$, $\omega_{3}=\sqrt{z_{3}}$. 
According to (27), we have

$$
\tau_{k}^{(j)}=\frac{1}{\omega_{k}}\left\{\arcsin \frac{\omega_{k}^{3}+\alpha_{2} \beta_{1} \omega_{k}}{b\left(\omega_{k}^{2}+\beta_{1}^{2}\right)}+2 j \pi\right\},
$$

where $k=1,2,3$ and $j=0,1, \ldots$, then $\pm i \omega_{k}$ is a pair of imaginary roots of Eq. (25) with $\tau_{k}^{(j)}$.

Define

$$
\tau_{0}=\tau_{k_{0}}^{(0)}=\min _{k=1,2,3}\left\{\tau_{k}^{(0)}\right\}, \quad \omega_{0}=\omega_{k_{0}}
$$

In order to investigate the distribution of the roots of Eq. (25), we need to introduce the following lemma [11].

Lemma 2 Consider the exponential polynomial

$$
\begin{aligned}
P\left(\lambda, e^{-\lambda \tau_{1}}, \ldots, e^{-\lambda \tau_{m}}\right)= & \lambda^{n}+p_{1}^{(0)} \lambda^{n-1}+\cdots+p_{n-1}^{(0)} \lambda+p_{n}^{(0)} \\
= & {\left[p_{1}^{(1)} \lambda^{n-1}+\cdots+p_{n-1}^{(1)} \lambda+p_{n}^{(1)}\right] e^{-\lambda \tau_{1}}+\cdots } \\
& +\left[p_{1}^{(m)} \lambda^{n-1}+\cdots+p_{n-1}^{(m)} \lambda+p_{n}^{(m)}\right] e^{-\lambda \tau_{m}},
\end{aligned}
$$

where $\tau_{i} \geq 0, i=1,2, \ldots, m$, and $p_{j}^{(i)}(i=0,1, \ldots, m ; j=1,2, \ldots, n)$ are constants. As $\left(\tau_{1}, \tau_{2}, \ldots, \tau_{m}\right)$ vary, the sum of the order of the zeros of $P\left(\lambda, e^{-\lambda \tau_{1}}, \ldots, e^{-\lambda \tau_{m}}\right)$ on the open right half plane can change if and only if there are zeros crossing the imaginary axis.

Let $\lambda(\tau)=\alpha(\tau)+i \omega(\tau)$ be the root of Eq. (25) near $\tau=\tau_{k}^{(j)}$ satisfying

$$
\alpha\left(\tau_{k}^{(j)}\right)=0, \quad \omega\left(\tau_{k}^{(j)}\right)=\omega_{k}
$$

Then the following transversality condition holds.

Lemma 3 Suppose that $z_{k}=\omega_{k}^{2}$ and $G^{\prime}\left(z_{k}\right) \neq 0$, then $\frac{\operatorname{Re} \lambda\left(\tau_{k}^{(j)}\right)}{d \tau}$ has the same sign as $G^{\prime}\left(z_{k}\right)$.

Proof Substituting $\lambda(\tau)$ into Eq. (25) and taking the derivative with respect to $\tau$, we obtain

$$
\left\{3 \lambda^{2}+2 \alpha_{1} \lambda+\alpha_{2}+b e^{-\lambda \tau}\left[1-\tau\left(\lambda+\beta_{1}\right)\right]\right\} \frac{d \lambda}{d \tau}=b \lambda e^{-\lambda \tau}\left(\lambda+\beta_{1}\right)
$$

Therefore,

$$
\left[\frac{d \lambda}{d \tau}\right]^{-1}=\frac{\left(3 \lambda^{2}+2 \alpha_{1} \lambda+\alpha_{2}\right) e^{\lambda \tau}}{b \lambda\left(\lambda+\beta_{1}\right)}+\frac{1}{\lambda\left(\lambda+\beta_{1}\right)}-\frac{\tau}{\lambda} .
$$

When $\tau=\tau_{k}^{(j)}, \lambda=i \omega_{k}, k=1,2,3$, we have

$$
\begin{aligned}
& {\left[\left(3 \lambda^{2}+2 \alpha_{1} \lambda+\alpha_{2}\right) e^{\lambda \tau}\right]_{\tau=\tau_{k}^{(j)}}=} \\
& \left(\left(\alpha_{2}-3 \omega_{k}^{2}\right) \cos \left(\omega_{k} \tau_{k}^{(j)}\right)-2 \alpha_{1} \omega_{k} \sin \left(\omega_{k} \tau_{k}^{(j)}\right)\right) \\
& +\left(2 \alpha_{1} \omega_{k} \cos \left(\omega_{k} \tau_{k}^{(j)}\right)+\left(\alpha_{2}-3 \omega_{k}^{2}\right) \sin \left(\omega_{k} \tau_{k}^{(j)}\right)\right) i \\
& {\left[\lambda\left(\lambda+\beta_{1}\right)\right]_{\tau=\tau_{k}^{(j)}}=-\omega_{k}^{2}+i \beta_{1} \omega_{k} .}
\end{aligned}
$$


According to (29), (32) and (33), we have

$$
\begin{aligned}
{\left[\frac{d \operatorname{Re}(\lambda)}{d \tau}\right]_{\tau=\tau_{k}^{(j)}}^{-1}=} & \operatorname{Re}\left[\frac{\left(3 \lambda^{2}+2 \alpha_{1} \lambda+\alpha_{2}\right) e^{\lambda \tau}}{b \lambda\left(\lambda+\beta_{1}\right)}\right]_{\tau=\tau_{k}^{(j)}}+\operatorname{Re}\left[\frac{b}{b \lambda\left(\lambda+\beta_{1}\right)}\right]_{\tau=\tau_{k}^{(j)}} \\
= & \frac{1}{\Lambda}\left\{\left[3 \omega_{k}^{4}+\omega_{k}^{2}\left(2 \alpha_{1} \beta_{1}-\alpha_{2}\right)\right] \cos \left(\omega_{k} \tau_{k}^{(j)}\right)\right. \\
& \left.+\left[\omega_{k}^{3}\left(2 \alpha_{1}-3 \beta_{1}\right)+\alpha_{2} \beta_{1} \omega_{k}\right] \sin \left(\omega_{k} \tau_{k}^{(j)}\right)-b^{2} \omega_{k}^{2}\right\} \\
= & \frac{1}{\Lambda}\left\{3 \omega_{k}^{6}+2\left(\alpha_{1}^{2}-2 \alpha_{2}\right) \omega_{k}^{4}+\left(\alpha_{2}^{2}-b^{2}\right) \omega_{k}^{2}\right\} \\
= & {\left[\frac{z_{k}\left(3 z_{k}^{2}+2 e_{1} z_{k}+e_{2}\right)}{\Lambda}\right]_{\tau=\tau_{k}^{(j)}}^{-1}=\frac{z_{k} G^{\prime}\left(z_{k}\right)}{\Lambda} }
\end{aligned}
$$

where $\Lambda=b^{2} \omega_{k}^{2}\left(\omega_{k}^{2}+\beta_{1}^{2}\right)^{2}>0$. Therefore,

$$
\operatorname{sign}\left[\frac{\operatorname{Re} d\left(\lambda\left(\tau_{k}^{(j)}\right)\right)}{d \tau}\right]=\operatorname{sign}\left[\frac{\operatorname{Re} d\left(\lambda\left(\tau_{k}^{(j)}\right)\right)}{d \tau}\right]^{-1}=\operatorname{sign} \frac{z_{k} G^{\prime}\left(z_{k}\right)}{\Lambda} \neq 0 .
$$

Now we apply the Hopf bifurcation theorem for functional differential equations [12] and obtain the following results.

Theorem 3 Suppose that $\left(\mathrm{H}_{1}\right)$ holds. Then

(i) when $\tau \in\left[0, \tau_{0}\right)$, the equilibrium $E_{1}^{*}$ of system $(4)_{0}$ is locally asymptotically stable,

(ii) when $\tau>\tau_{0}$, the equilibrium $E_{1}^{*}$ of system $(4)_{0}$ is unstable,

(iii) when $\tau=\tau_{0}$, system $(4)_{0}$ undergoes Hopf bifurcation at $E_{1}^{*}$.

(2) $E^{*}=E_{2}^{*}$, or $E_{3}^{*}$

When $E^{*}=E_{2}^{*}$, or $E_{3}^{*}$, the coefficients of Eq. (25) are $\alpha_{1}=1-2 a c, \alpha_{2}=-2 a c-a, \beta_{1}=-2 a c$. Under condition $\left(\mathrm{H}_{1}\right)$, Eq. (25) has at least one positive real root, so $E_{2}^{*}$ and $E_{3}^{*}$ are unstable equilibria.

From above, we have obtained the conditions under which $E_{1}^{*}$ undergoes Hopf bifurcation at $\tau=\tau_{0}$. Now, we will derive the explicit formulae determining the direction and the stability of the periodic solutions bifurcating from the equilibrium $E_{1}^{*}$. Let $\tau=\tau_{0}+\mu$, $t=\tau \bar{t}$ and omit ' - ' above $t$, we rewrite $(4)_{\varepsilon}$ as

$$
\dot{u}(t)=L_{\mu} u_{t}+f\left(\mu, u_{t}\right)+\varepsilon P_{T} \Phi(\phi),
$$

where

$$
\begin{aligned}
& L_{\mu} \phi=\left(\tau_{0}+\mu\right) A\left[\begin{array}{l}
\phi_{1}(0) \\
\phi_{2}(0) \\
\phi_{3}(0)
\end{array}\right]+\left(\tau_{0}+\mu\right) B\left[\begin{array}{l}
\phi_{1}(-1) \\
\phi_{2}(-1) \\
\phi_{3}(-1)
\end{array}\right], \\
& f_{\mu}(\phi)=\left(\tau_{0}+\mu\right)\left[\begin{array}{c}
-a d \phi_{1}^{3}(0) \\
0 \\
0
\end{array}\right], \quad \Phi(\phi)=\left(\tau_{0}+\mu\right)\left[\begin{array}{c}
0 \\
\phi_{2}(0) \\
0
\end{array}\right],
\end{aligned}
$$


$A, B$ are as in (24). We write $L_{\mu} \phi$ as the form $L_{\mu} \phi=\int_{-1}^{0} d \eta(\theta, \mu) \phi(\mu)$, where

$$
d \eta(\theta, \mu)=\left(\tau_{0}+\mu\right) A \delta(\theta)+\left(\tau_{0}+\mu\right) B \delta(\theta+1)
$$

$\delta(\theta)$ is the Dirac delta function. Then Eq. $(4)_{\varepsilon}$ can be written as

$$
\dot{u}(t)=A(\mu) u_{t}+R u_{t},
$$

where

$$
A(\mu) \phi= \begin{cases}\frac{d \phi(\theta)}{d \theta}, & \theta \in[-1,0), \\ \int_{-1}^{0} d \eta(s, \mu) \phi(s), & \theta=0,\end{cases}
$$

and

$$
R \phi= \begin{cases}0, & \theta \in[-1,0), \\ f_{\mu}(\phi)+\varepsilon P_{T} \Phi(\phi), & \theta=0 .\end{cases}
$$

Assume that $q(\theta)$ is the eigenvector of $A(0)$ corresponding to $i \omega_{0} \tau_{0}$, then $A(0) q(\theta)=$ $i \tau_{0} \omega_{0} q(\theta)$. It follows from the definition of $A(0)$ that

$$
\left(\begin{array}{ccc}
i \omega_{0}+a c & -a & 0 \\
-1 & i \omega_{0}+1 & -1 \\
0 & b e^{-i \omega_{0} \tau_{0}} & i \omega_{0}
\end{array}\right) q(\theta)=\left(\begin{array}{l}
0 \\
0 \\
0
\end{array}\right)
$$

Thus, we can easily compute $q(\theta)=(1, \alpha, \beta) e^{i \omega_{0} \tau_{0} \theta}$, where

$$
\alpha=\frac{a c+i \omega_{0}}{a}, \quad \beta=\frac{b e^{-i \omega_{0} \tau_{0}}\left(-\omega_{0}+i a c\right)}{a \omega_{0}} .
$$

We can verify that $q^{*}(\theta)=D\left(1, \alpha^{*}, \beta^{*}\right) e^{i \omega_{0} \tau_{0} \theta}$ is the eigenvector of $A^{*}$ corresponding to $-i \omega_{0} \tau_{0}$, where

$$
\alpha^{*}=a c-i \omega_{0}, \quad \beta^{*}=\frac{\omega_{0}+i a c}{\omega_{0}} .
$$

In order to assure the bilinear inner product $\left\langle q^{*}(\theta), q(\theta)\right\rangle=1$, we have

$$
\begin{aligned}
& \left\langle q^{*}(\theta), q(\theta)\right\rangle \\
& \quad=\bar{D}\left(1, \bar{\alpha}^{*}, \bar{\beta}^{*}\right)(1, \alpha, \beta)^{T}-\int_{-1}^{0} \int_{\xi=0}^{\theta} \bar{D}\left(1, \bar{\alpha}^{*}, \bar{\beta}^{*}\right) e^{-i(\xi-\theta) \omega_{0} \tau_{0}} d \eta(\theta)(1, \alpha, \beta)^{T} e^{i \xi \omega_{0} \tau_{0}} d \xi \\
& \quad=\bar{D}\left(1+\alpha \bar{\alpha}^{*}+\beta \bar{\beta}^{*}-\int_{-1}^{0}\left(1, \bar{\alpha}^{*}, \bar{\beta}^{*}\right) \theta e^{i \theta \omega_{0} \tau_{0}} d \eta(\theta)(1, \alpha, \beta)^{T}\right) \\
& =\bar{D}\left(1+\alpha \bar{\alpha}^{*}+\beta \bar{\beta}^{*}+b \alpha \bar{\beta}^{*} \tau_{0} e^{-i \omega_{0} \tau_{0}}\right) .
\end{aligned}
$$

Therefore, we can choose $\bar{D}$ as

$$
\bar{D}=\left[1+\alpha \bar{\alpha}^{*}+\beta \bar{\beta}^{*}+b \alpha \bar{\beta}^{*} \tau_{0} e^{-i \omega_{0} \tau_{0}}\right]^{-1}=\frac{a \omega_{0}^{2}}{a_{1}+i a_{2}+b e^{-i \omega_{0} \tau_{0}}\left(a_{3}+i a_{4}\right)}=\frac{a \omega_{0}^{2}\left(d_{1}-i d_{2}\right)}{d_{1}^{2}+d_{2}^{2}},
$$



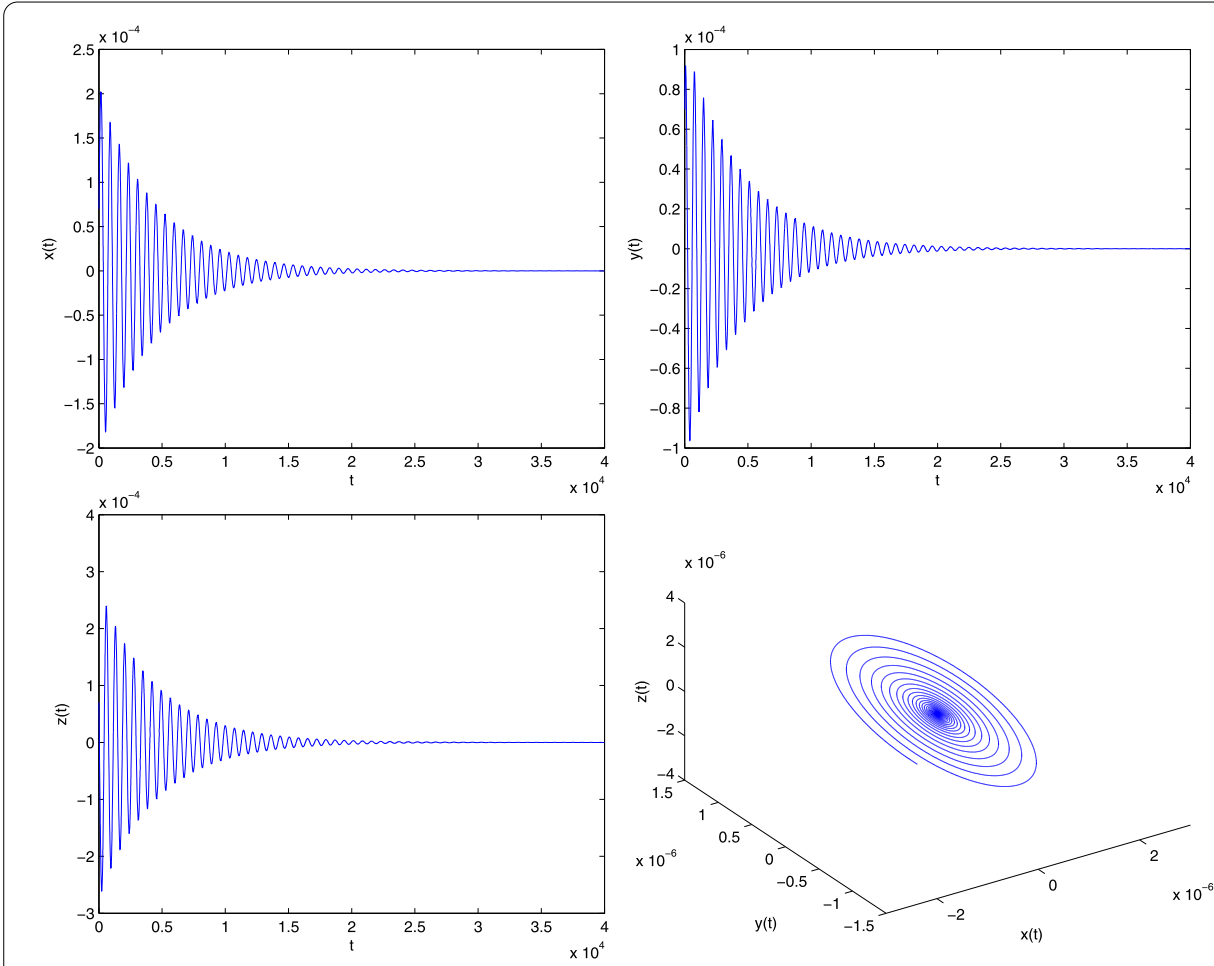

Figure 1 The trajectories and phase graphs of system (41) with $T=72.3, \varepsilon=0, \tau=0.01, E^{*}$ is asymptotically stable.

where $a_{1}=a \omega_{0}^{2}+a^{2} c^{2} \omega_{0}^{2}-\omega_{0}^{4}, a_{2}=2 a c \omega_{0}^{3}, a_{3}=a^{2} c^{2}-\omega_{0}^{2}+2 a c \tau \omega_{0}^{2}, a_{4}=2 a c \omega_{0}+\tau_{0} \omega_{0}^{3}-$ $\tau_{0} \omega_{0} a^{2} c^{2}, d_{1}=a_{1}+b a_{3} \cos \left(\omega_{0} \tau_{0}\right)+b a_{4} \sin \left(\omega_{0} \tau_{0}\right), d_{2}=a_{2}+b a_{4} \cos \left(\omega_{0} \tau_{0}\right)-b a_{3} \sin \left(\omega_{0} \tau_{0}\right)$.

We can get $\left\langle q^{*}(s), \bar{q}(\theta)\right\rangle=0$.

In what follows, we will obtain the coordinates to describe the center manifold $C_{0}$ at $\mu=0$. Notice that $u_{t}(\theta)=\left(x_{t}(\theta), y_{t}(\theta), z_{t}(\theta)\right)^{T}=z q(\theta)+\bar{z} \bar{q}(\theta)+W(t, \theta)$, then we have

$$
\begin{aligned}
x_{t}(0)= & z+\bar{z}+W_{20}^{(1)}(0) \frac{z^{2}}{2}+W_{11}^{(1)}(0) z \bar{z}+W_{02}^{(1)}(0) \frac{\bar{z}^{2}}{2} \\
& +W_{30}^{(1)}(0) \frac{z^{3}}{6}+W_{21}^{(1)}(0) \frac{z^{2} \bar{z}}{2}+\cdots, \\
y_{t}(0)= & z \alpha+\bar{z} \bar{\alpha}+W_{20}^{(2)}(0) \frac{z^{2}}{2}+W_{11}^{(2)}(0) z \bar{z}+W_{02}^{(2)}(0) \frac{\bar{z}^{2}}{2} \\
& +W_{30}^{(2)}(0) \frac{z^{3}}{6}+W_{21}^{(2)}(0) \frac{z^{2} \bar{z}}{2}+\cdots, \\
z_{t}(0)= & z \beta+\bar{z} \bar{\beta}+W_{20}^{(3)}(0) \frac{z^{2}}{2}+W_{11}^{(3)}(0) z \bar{z}+W_{02}^{(3)}(0) \frac{\bar{z}^{2}}{2} \\
& +W_{30}^{(3)}(0) \frac{z^{3}}{6}+W_{21}^{(3)}(0) \frac{z^{2} \bar{z}}{2}+\cdots .
\end{aligned}
$$

Thus, from Eq. (20) we have

$$
\bar{q}^{*}(0) f_{0}\left(u_{t}\right)=\bar{D} \tau_{0}\left(1, \bar{\alpha}^{*}, \bar{\beta}^{*}\right)\left(\begin{array}{c}
-a d x_{t}^{3}(0) \\
0 \\
0
\end{array}\right)=-a d \tau_{0} \bar{D} x_{t}^{3}(0),
$$




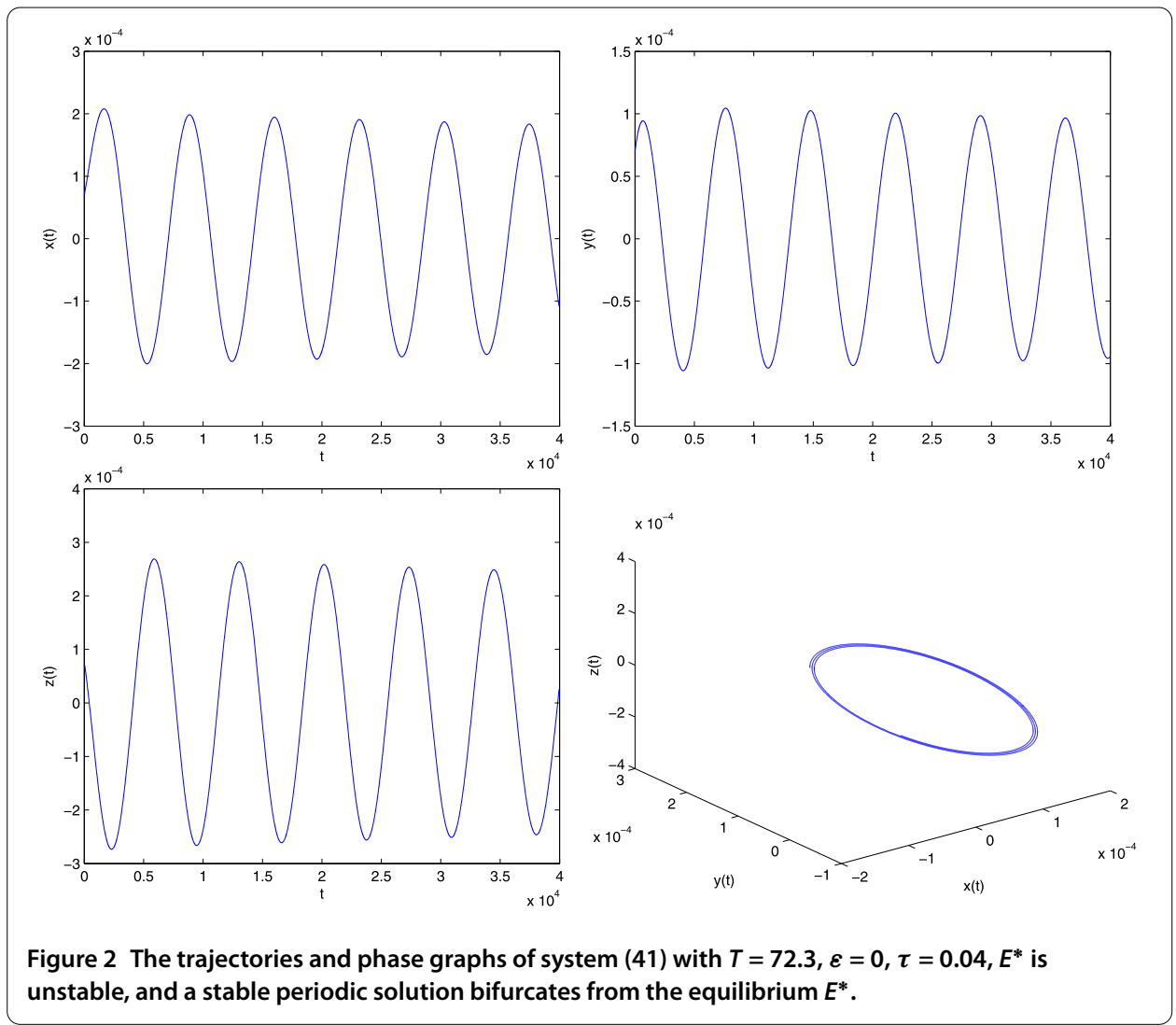

then

$$
g_{20}=g_{11}=g_{02}=0, \quad g_{21}=-6 a d \tau_{0} \bar{D} .
$$

Thus, we can compute the following values:

$$
c_{1}(0)=\frac{g_{21}}{2}, \quad \mu_{2}=-\frac{\operatorname{Re}\left\{c_{1}(0)\right\}}{\operatorname{Re}\left\{\lambda^{\prime}\left(\tau_{0}\right)\right\}}, \quad \beta_{2}=2 \operatorname{Re}\left\{c_{1}(0)\right\}
$$

which determine the quantities of bifurcating periodic solutions in the center manifold at the critical value $\tau_{0}$, i.e., $\mu_{2}$ determines the directions of a Hopf bifurcation: if $\operatorname{Re}\left\{\lambda^{\prime}\left(\tau_{0}\right)\right\}>0, \mu_{2}>0$ (resp. $\mu_{2}<0$ ), then the Hopf bifurcation is supercritical (resp. subcritical) and the periodic solutions exist for $\tau>\tau_{0}\left(\tau<\tau_{0}\right)$. If $\operatorname{Re}\left\{\lambda^{\prime}\left(\tau_{0}\right)\right\}<0$, however, the bifurcating periodic solutions are on the opposite direction. $\beta_{2}$ determines the stability of the bifurcation periodic solutions: the bifurcating periodic solutions are stable (unstable) if $\beta_{2}<0\left(\beta_{2}>0\right)$. Furthermore, we can get $E(0)=\frac{3 a^{2} d \tau_{0} \omega_{0}^{2} d_{1}}{d_{1}^{2}+d_{2}^{2}}$, and $F(0)=\frac{3 a^{2} d \tau_{0} \omega_{0}^{2} d_{2}}{d_{1}^{2}+d_{2}^{2}}$. Let $W=0$, from Eq. (20) and Eq. (22), we have

$$
\begin{aligned}
\Psi_{x}(x, y) & =\operatorname{Re}\left\{\bar{q}^{*}(0) \Phi(2 \operatorname{Re}(x+i y) q(0))\right\} \\
& =\operatorname{Re}\left\{\bar{D} \tau_{0}\left(1, \bar{\alpha}^{*}, \bar{\beta}^{*}\right)\left(\begin{array}{c}
0 \\
(x+i y) \alpha+(x-i y) \bar{\alpha} \\
0
\end{array}\right)\right\}
\end{aligned}
$$



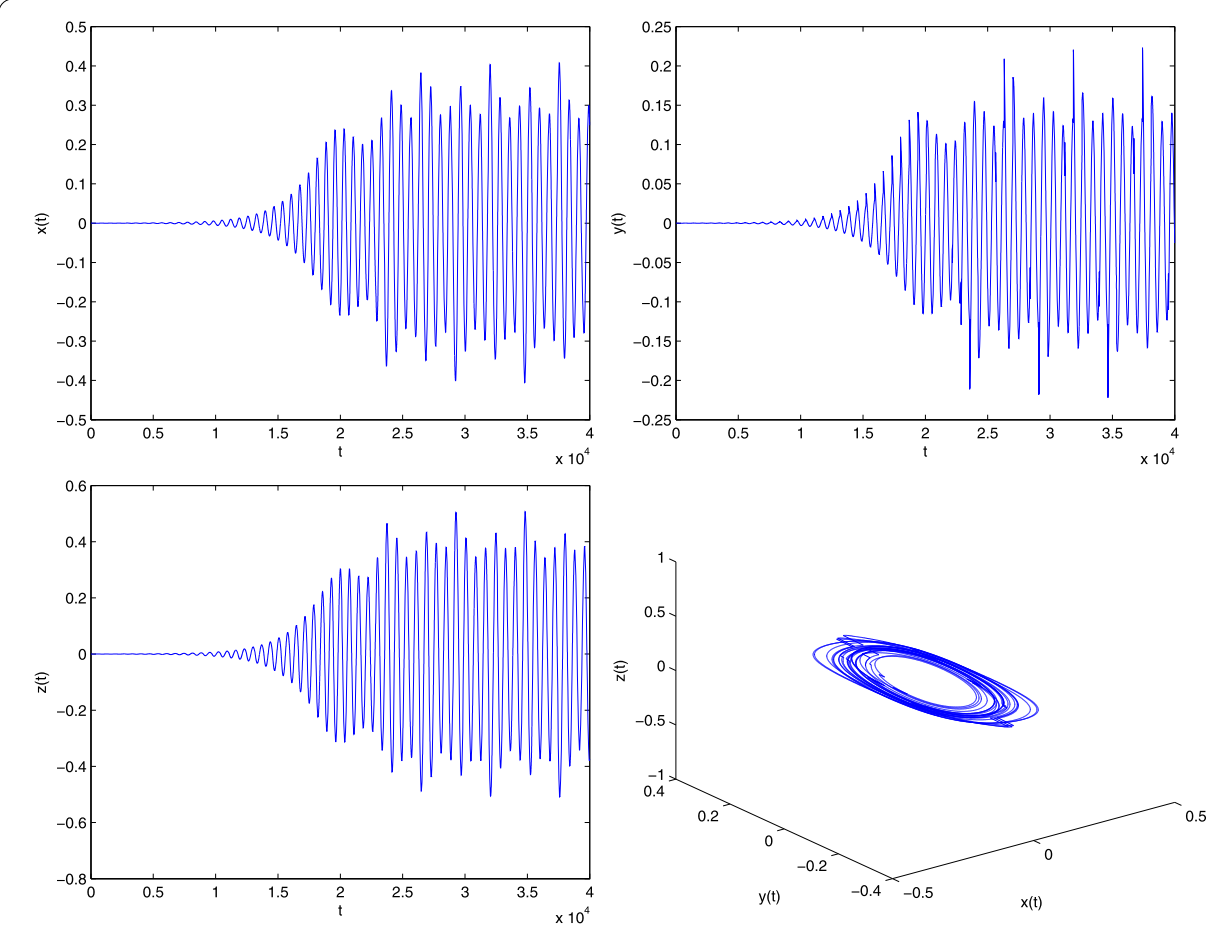

Figure 3 The trajectories and phase graphs of system (41) with $T=69.3, \varepsilon=0.55, \tau=0.04$, a rank one strange attractor occurs.

$$
\begin{aligned}
& =\operatorname{Re}\left\{\bar{D} \tau_{0} \bar{\alpha}^{*}((x+i y) \alpha+(x-i y) \bar{\alpha})\right\} \\
& =2 \tau_{0} \omega_{0}^{2}\left(a c x-\omega_{0} y\right) \frac{d_{3}}{d_{1}^{2}+d_{2}^{2}}, \\
\Psi_{y}(x, y) & =\operatorname{Im}\left\{\bar{q}^{*}(0) \Phi(2 \operatorname{Re} z q(0))\right\}=2 \tau_{0} \omega_{0}^{2}\left(a c x-\omega_{0} y\right) \frac{d_{4}}{d_{1}^{2}+d_{2}^{2}},
\end{aligned}
$$

where $d_{3}=a c d_{1}+\omega_{0} d_{2}, d_{4}=\omega_{0} d_{1}-a c d_{2}$. Let $x=\cos \theta, y=\sin \theta$, then

$$
\begin{aligned}
\phi(\theta) & =\cos \theta \Psi_{x}\left(\hat{s}_{0}\right)+\sin \theta \Psi_{y}\left(\hat{s}_{0}\right) \\
& =\frac{2 \tau_{0} \omega_{0}^{2}}{d_{1}^{2}+d_{2}^{2}}\left[a c d_{3} \cos ^{2} \theta+\left(a c d_{4}-\omega_{0} d_{3}\right) \sin \theta \cos \theta-\omega_{0} d_{4} \sin ^{2} \theta\right] .
\end{aligned}
$$

Thus, if $3 a^{2} d \tau_{0} \omega_{0}^{2} d_{1}>0$, then we have $E(0)>0$ and $\phi(\theta)$ is easy to be verified to be a Morse function. So, according to Theorem 2, there is a constant $K_{2}$ such that if $\left|\varepsilon \frac{F(0)}{E(0)}\right|>K_{2}$, we can get an observable rank one chaos.

\section{Numerical simulations}

Now we move to the parameters for which rank one attractors are observable. We consider the following system:

$$
\left\{\begin{array}{l}
\dot{x}(t)=1.9\left(y(t)-0.2495 x(t)+x^{3}(t)\right), \\
\dot{y}(t)=x(t)-y(t)+z(t)+\varepsilon P_{T} y(t), \\
\dot{z}(t)=-2.249999 y(t-\tau) .
\end{array}\right.
$$



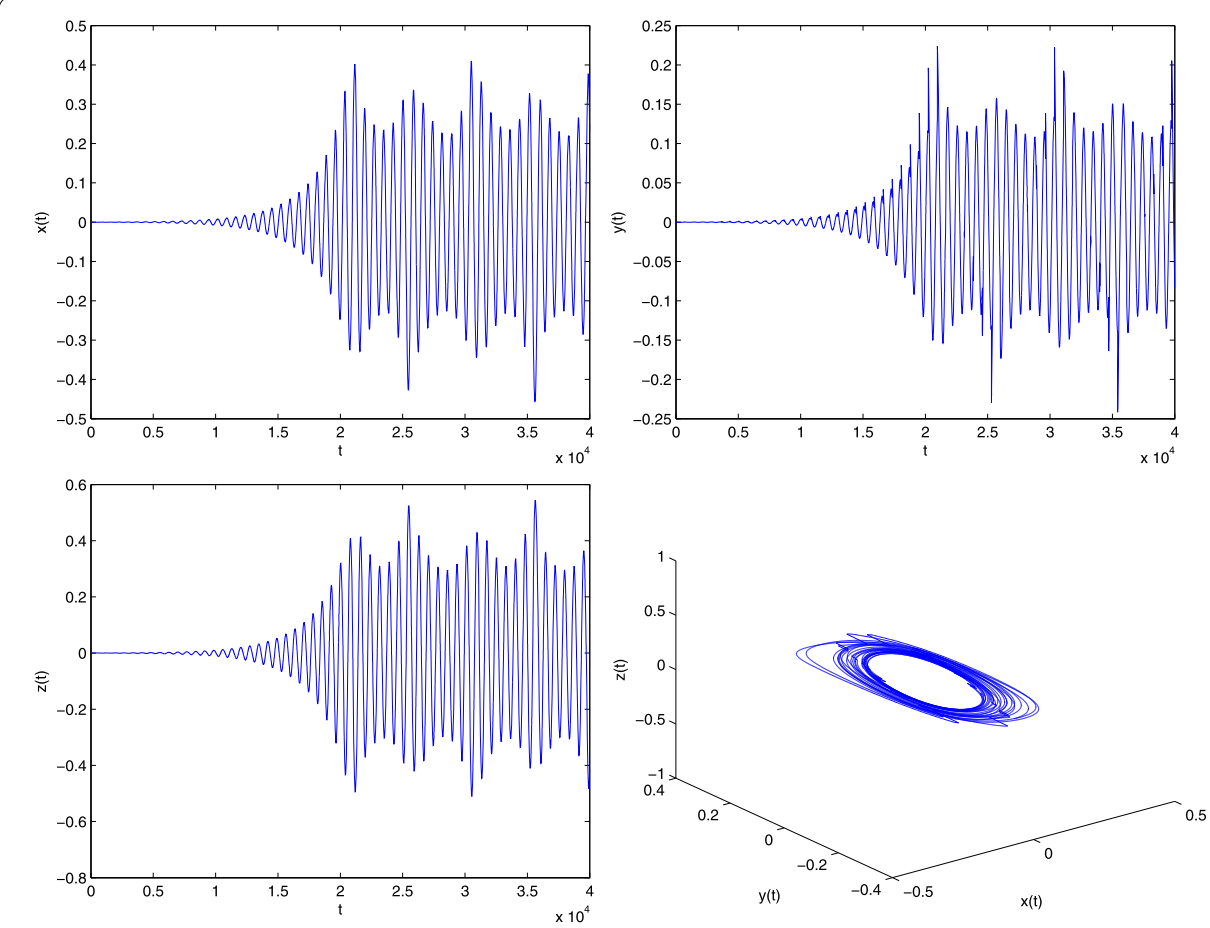

Figure 4 The trajectories and phase graphs of system (41) with $T=72.3, \varepsilon=0.55, \tau=0.04$, a rank one strange attractor occurs.
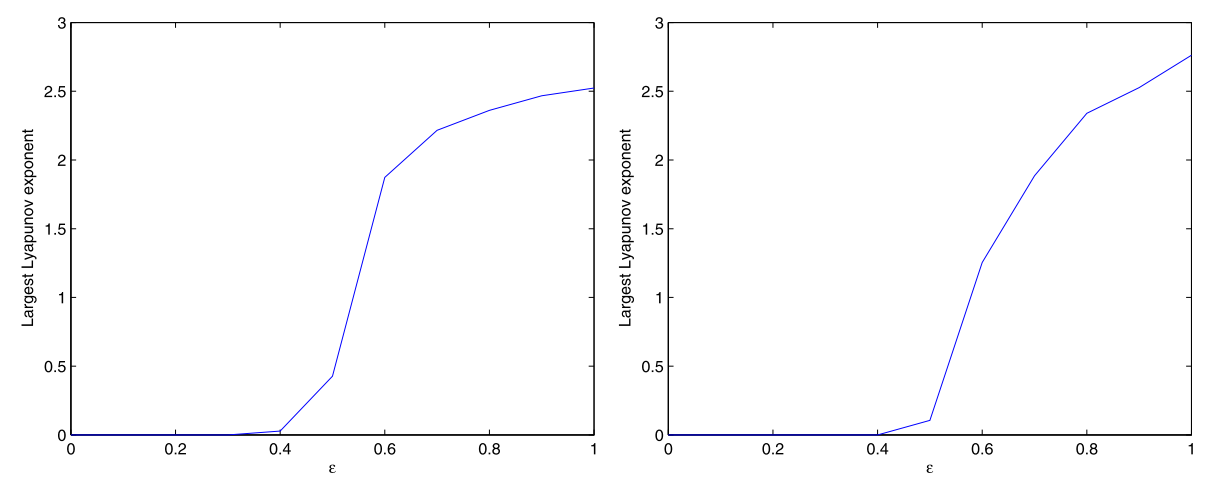

Figure 5 Largest Lyapunov exponents $\lambda$ versus $\varepsilon$ for system (41) with $T=69.3$ (left), $T=72.3$ (right), $\tau=0.04$ and $\varepsilon$ varying from 0 to 1.0 .

From the discussion of Section 4, we have $\omega_{0} \doteq 0.8805, \tau_{0} \doteq 0.04, \operatorname{Re}\left\{\lambda^{\prime}(0)\right\} \doteq 1.2968$, $\operatorname{Re}\left\{c_{1}(0)\right\} \doteq-0.0024, \mu_{2} \doteq 0.0018, \beta_{2} \doteq-0.0047, T_{2} \doteq 3.6421$, so we know that the equilibrium $E_{1}^{*}$ is asymptotically stable when $\varepsilon=0$ and $\tau \in[0,0.04$ ) (see Figure 1). Further, we know that the bifurcation is supercritical and the stable periodic solution emerges from the equilibrium $E_{1}^{*}$ (see Figure 2).

We also get $E(0) \doteq 0.0024>0,\left|\frac{F(0)}{E(0)}\right| \doteq 59.7693$. We choose $T$ in interval $(69,73)$, which is quite 'large' to afford a long relaxation period between consecutive kicks of the external force, and choose $\varepsilon$ in $(0,1)$. In Figure 3, we present a rank one strange attractor at $(\tau, \varepsilon, T)=(0.04,0.55,69.3)$. In Figure 4, a rank one strange attractor at $(\tau, \varepsilon, T)=$ 
$(0.04,0.55,72.3)$ is shown. In Figure 5 , the largest Lyapunov exponents $\lambda$ versus $\varepsilon$ for system (41) with $T=69.3$ and $T=72.3$ are given. The results of our numerical simulations are in perfect agreement with the predictions of the rank one theory in Section 3.

\section{Conclusions}

We have developed rank one theory from an ordinary differential equation to a timedelayed system and considered the existence of rank one chaos in time-delayed Chua's system. It is shown that rank one strange attractors occur when the delayed system under a periodic kick undergoes supercritical Hopf bifurcation. We also show some results of numerical simulations to support the rank one theory.

\section{Competing interests}

The authors declare that they have no competing interests.

\section{Authors' contributions}

All authors contributed equally to the writing of this paper. All authors read and approved the final manuscript.

\section{Author details}

'Department of Applied Mathematics, Kunming University of Science and Technology, Kunming, Yunnan 650500, P.R. China. ${ }^{2}$ Department of Mathematics, Qingdaobinhai University, Qingdao, Shandong 266555, P.R. China.

\section{Acknowledgements}

This research is supported by the National Natural Science Foundation of China (Nos. 11061016, 11461036).

Received: 10 October 2014 Accepted: 30 January 2015 Published online: 04 March 2015

\section{References}

1. Wang, Q, Oksasoglu, A: Strange attractors with one direction of instability. Commun. Math. Phys. 218, 1-97 (2001)

2. Wang, $Q$, Young, L: Toward a theory of rank one attractors. Ann. Math. 167(2), 349-480 (2008)

3. Chen, F, Han, M: Rank one chaos in a class of planar systems with heteroclinic cycle. Chaos 19(4), 043122 (2009)

4. Fang, $\mathrm{H}$ : Synchronization of two rank-one chaotic systems without and with delay via linear delayed feedback control. J. Appl. Math. 2012, Article ID 325131 (2012)

5. Jackson, L, Lindgren, A, Kim, Y: A chaotic attractor from Chua's circuit. IEEE Trans. Circuits Syst. 31(12), 1055-1058 (1984)

6. Wang, X, Zhong, G, Tang, K, et al.: Generating chaos in Chua's circuit via time-delay feedback. IEEE Trans. Circuits Syst. I, Fundam. Theory Appl. 48(9), 1151-1156 (2001)

7. Wang, Q, Oksasoglu, A: Strange attractors in periodically kicked Chua's circuit. Int. J. Bifurc. Chaos 15(1), $83-98$ (2005)

8. Young, L: What are SRB measures, and which systems have them? J. Stat. Phys. 108, 733-754 (2002)

9. Wang, Q, Oksasoglu, A: Rank one chaos: theory and applications. Int. J. Bifurc. Chaos 185, 1261-1319 (2008)

10. Hassard, B, Kazarinoff, D, Wan, Y: Theory and Applications of Hopf Bifurcation. Cambridge University Press, Cambridge (1981)

11. Ruan, S, Wei, J: On the zeros of transcendental functions with applications to stability of delay differential equations with two delays. Dyn. Contin. Discrete Impuls. Syst., Ser. A Math. Anal. 10, 863-874 (2003)

12. Chow, S, Hale, J: Methods of Bifurcation Theory. Springer, Berlin (1981)

\section{Submit your manuscript to a SpringerOpen ${ }^{\ominus}$ journal and benefit from:}

- Convenient online submission

Rigorous peer review

- Immediate publication on acceptance

- Open access: articles freely available online

- High visibility within the field

- Retaining the copyright to your article 